\title{
Investigation of Dynamic and Static Recrystallization Behavior During Thermomechanical Processing in API-X70 Microalloyed Steel
}

\author{
Bahman Mirzakhani, Hossein Arabi, Mohammad Taghi Salehi, Shahin Khoddam,
} Seyed Hossein Seyedein, and Mohammad Reza Aboutalebi

\section{Erratum to: Journal of Materials Engineering and Performance DOI 10.1007/s11665-008-9338-x}

The correct title of the paper and authors' list are as follows: Investigation of Dynamic and Static Recrystallization Behavior During Thermomechanical Processing in API-X70 Microalloyed Steel

Bahman Mirzakhani ${ }^{\mathrm{a}}$, Hossein Arabi ${ }^{\mathrm{b}}$, Mohammad Taghi Salehi $^{\mathrm{c}}$, Shahin Khoddam ${ }^{\mathrm{d}}$, Seyed Hossein Seyedein ${ }^{\mathrm{e}}$, Mohammad Reza Aboutalebi ${ }^{\mathrm{f}}$

${ }^{a}$ Department of Metallurgical Engineering, Iran University of Science and Technology, P.O. Box 16844-13114, Tehran, Iran

E-mail: bmirzakhani@iust.ac.ir

${ }^{\mathrm{b}}$ Department of Metallurgical Engineering, Iran University of Science and Technology, P.O. Box 16844-13114, Tehran, Iran

E-mail: arabi@iust.ac.ir
The online version of the original article can be found under doi:10.1007/s11665-008-9338-x.

Bahman Mirzakhani, Hossein Arabi, Mohammad Taghi Salehi, Seyed Hossein Seyedein, and Mohammad Reza Aboutalebi, Department of Metallurgical Engineering, Iran University of Science and Technology, P.O. Box 16844-13114, Tehran, Iran; and Shahin Khoddam, Department of Mechanical Engineering, Monash University, Clayton Campus, Melbourne, Victoria 3800, Australia. Contact e-mails: bmirzakhani@iust.ac.ir, arabi@iust.ac.ir, salehi@iust. ac.ir, shahin.khoddam@eng.monash.edu.au, seyedein@iust.ac.ir, mrezab@ iust.ac.ir.
${ }^{\mathrm{c}}$ Department of Metallurgical Engineering, Iran University of Science and Technology, P.O. Box 16844-13114, Tehran, Iran

E-mail: salehi@iust.ac.ir

${ }^{\mathrm{d}}$ Department of Mechanical Engineering, Monash University, Clayton Campus, Vic 3800, Australia

E-mail: shahin.khoddam@eng.monash.edu.au

e Department of Metallurgical Engineering, Iran University of Science and Technology, P.O. Box 16844-13114, Tehran, Iran

E-mail: seyedein@iust.ac.ir

${ }^{\mathrm{f}}$ Department of Metallurgical Engineering, Iran University of Science and Technology, P.O. Box 16844-13114, Tehran, Iran

E-mail: mrezab@iust.ac.ir 